\title{
Selection of Patients with Colorectal/Liver Metastases for Surgical Intervention: Current Issues and Challenges
}

\author{
Kevin N. Shah • Bryan M. Clary
}

Published online: 1 July 2014

(C) Springer Science + Business Media New York 2014

\begin{abstract}
Resection of colorectal liver metastases (CRLM) represents the best chance for cure, but appropriate selection of patients can present challenging clinical scenarios. The PubMed database was searched for articles relevant to CRLM published between 1990 and December 2013. The ability to safely achieve curative resection depends heavily on the adequacy of the future liver remnant (FLR). The FLR volume can be improved through several techniques including portal vein embolization and surgical portal vein ligation with in situ liver split (ALPPS). Disappearing liver metastases (DLM) may develop as a result of robust response to chemotherapy. Concordance between radiographic DLM and true pathologic response is poor and up to $85 \%$ of DLM that undergo resection will demonstrate viable tumor on pathological examination. The rate of true pathologic response for lesions treated with hepatic artery infusion may be higher. Modern chemotherapeutics and techniques to improve the FLR have increased the number of patients who are candidates for potentially curative resection. In cases of DLM, curative resection should aim to include all sites of disease present prior to systemic chemotherapy if possible.
\end{abstract}

Keywords Colorectal cancer - Liver metastases . Disappearing liver metastases · Portal vein embolization . ALPPS

This article is part of the Topical Collection on Colorectal Liver Metastases.

K. N. Shah · B. M. Clary $(\bowtie)$

Division of Surgical Oncology, Duke University Medical Center,

10 Searle Drive, 485 Seeley Mudd Building, DUMC 3247,

Durham, NC 27710, USA

e-mail: bryan.clary@dm.duke.edu

K. N. Shah

e-mail: kevin.n.shah@dm.duke.edu

\section{Introduction}

The development of liver metastases occurs in over $50 \%$ of patients with colorectal cancer [1]. The presence of colorectal liver metastases (CRLM) was once viewed with a uniformly grim outlook, but modern advances in anesthesia, imaging, surgical technique, and chemotherapeutics have allowed an increasing number of patients to become candidates for potentially curative resection. Central to the expansion of partial hepatectomy as a treatment option for CRLM has been the realization that bilaterality, multiplicity, limited margins, and even limited extrahepatic disease should not preclude consideration of this treatment modality. The significant improvements in outcomes with contemporary chemotherapy regimens in unresectable patients have led to their routine adoption in patients with resectable/resected disease as well. The improved response rates with these regimens in the context of liberalized definitions of resectability have allowed application of partial hepatectomy to intrahepatic distributions of disease that heretofore were not feasible and/or advisable. For patients with initially unresectable disease distributions, approximately $50 \%$ demonstrate radiologic responses and $20 \%$ will ultimately become candidates for curative resections [2].

As a consequence of these contemporary trends, clinicians have had to focus on a new set of issues that revolve around the quality of the future liver remnant (FLR). These include hepatotoxicity and increased perioperative morbidity associated with pre-hepatectomy chemotherapy, the role of pre-hepatectomy adjunctive treatments is to enhance the FLR and parenchymal preservation strategies. These advances have brought greater complexity to the decisions regarding the proper timing of multimodality therapies including hepatic surgery, primary tumor 
extirpation, systemic therapy, and radiation (for rectal cancer). Lastly, the increased efficacy of modern chemotherapy has also resulted in another challenge; how to treat lesions that disappear after extended treatment with chemotherapy. The natural history of these disappearing liver metastases (DLM) is often difficult to predict and there is some controversy as to what the most appropriate treatment should entail.

It is the purpose of the review to discuss some of the current challenges associated with the expanding indications for resection and in the multimodality therapy. Specifically, we will focus on preoperative and intraoperative strategies to optimize the FLR and address the problem of DLM.

\section{Optimization of the Liver Remnant}

To be considered resectable, patients with CRLM (who are appropriate medical candidates) must in general meet the following criteria at a minimum: (1) An ability to obtain complete (R0) resection of the primary tumor as well as intra- and extrahepatic (limited) metastases; (2) The presence of 2 contiguous segments in the remnant liver with intact vascular inflow/outflow and intact biliary drainage; (3) Sufficient liver volume must remain after resection. This final requirement generally correlates to minimum an FLR volume (FLV) equivalent to $20 \%$ of the total estimated liver volume for those with normal liver parenchyma, $30 \%$ for those with steatosis/steatohepatitis (particularly in the context of chemotherapy associated liver injury), and $40 \%$ in cirrhotic patients [3].

In addressing increasing intrahepatic tumor multiplicity, bilateral disease, or lesions in difficult intrahepatic locations, there can be significant challenges in meeting the second and third criteria (adequate FLV and intact support structures). These criteria reflect the fact that the volume of the liver remnant and its underlying quality are the principal determinants of perioperative outcomes. Post hepatectomy liver failure remains a major source of morbidity and is the dominant factor in postoperative mortality. One of the keys to successful outcomes following partial hepatectomy lies in the preservation of parenchyma. In almost every study that has looked at predictors of perioperative outcomes following hepatectomy, there exist a few factors that are consistently relevant: the volume of the hepatectomy (and hence the volume of the remnant), the performance of concomitant major procedures, and the presence of intrinsic liver disease. As such attention to the status of the future liver remnant is fundamental and parenchymal preservation key. Several strategies have emerged to optimize FLV in patients with expected marginal liver remnants. These include portal vein embolization (PVE), staged hepatectomy, and ALPPS (Associating Liver Partition and Portal Vein ligation for Staged Hepatectomy).

\section{Portal Vein Embolization}

Portal vein embolization was first used in the 1980s to help extend the limits of resectability of primary hepatobiliary malignancies by causing contralateral hepatic hyperplasia and ipsilateral atrophy [4]. By accessing the portal vein percutaneously, the main portal vein branch contralateral to the proposed liver remnant (i.e., of the liver to be resected) is occluded using any one of a number of embolic materials including coils, gelatin, fibrin glue, cyanoacrylate, and polyvinyl alcohol [3]. Therefore, when a right hepatectomy is required to resect CRLM and the FLV is judged to be insufficient, the right portal vein can be embolized to encourage FLV hypertrophy. In cases where an extended right hepatectomy is necessary, investigators have advocated embolization of the right portal vein as well as the left medial portal vein branch (to segment IV) to further increase hyperplasia of segments II and III [5•]. It should be noted that in contrast to extended right hepatectomies (where resected volume is often greater than $80 \%$ ), left-sided resections including extended left hepatectomies generally leave sufficient FLV and only rarely require PVE. Exceptions to this include cases where right-sided wedge resections are necessary in conjunction with left hepatectomy, and patients who have underlying liver disease.

The maximal rate of liver regeneration occurs between 7 and 14 days in animal models and by about 3-4 weeks volume gains have generally been maximized. Rates of growth and the time required for liver regeneration are typically longer in those with underlying liver pathology and diabetics [4]. Keeping this in mind, a 3-4 week interval between PVE and hepatectomy is necessary to ensure that liver regeneration has been maximized. In individuals with normal regenerative capacity, increases in RLV of 20-38\% are achievable [6, 7]. Complications as a result of PVE occur in less than $5 \%$, and it is a generally well-tolerated and safe procedure [3]. Resection after PVE is also safe, and in patients with marginal FLV, preoperative PVE can reduce morbidity compared to those who are not embolized and are left with an insufficient RLV [8]. Just looking at the absolute size of the FLV, however, may be an insufficient predictor of perioperative outcomes. Vauthey and colleagues have suggested that the rate of hypertrophy (kinetic growth rate) is a better predictor of post-hepatectomy morbidity than current absolute volume measurements [9]. Additionally, PVE can be performed in the presence of ongoing systemic therapy which some have advocated as a means of minimizing the phenomenon of enhanced tumor growth that can occur in the milieu of the hypertrophying liver [10•]. 
Staged Resections

An alternative and often complimentary approach to PVE for patients with bilateral disease is the performance of staged hepatic resections [11]. The typical context in which this approach is utilized involves the need for a right hepatectomy in the presence of lesion(s) in the left liver that are amenable to wedge resections. Patients typically have marginally resectable disease for which pre-hepatectomy chemotherapy is required to facilitate a response. Although it is more common to perform the left liver wedges first and then prepare the right liver for resection with right portal vein embolization, this need not necessarily be the case. The rationale for such a strategy relates to the risk of tumor progression in the non-embolized left liver. Once the necessary remnant regeneration has occurred (about 4 weeks post-PVE), the second stage of right or extended right hepatectomy can be performed to clear the remaining metastases.

\section{ALPPS}

The technique of "Associating Liver Partition and Portal Vein ligation for Staged Hepatectomy" (ALPPS) has become a hotly debated topic among liver surgeons, and its role in optimizing FLV is controversial. Schnitzbauer et al. [12•] published their initial experience and technique in 2012. The first stage of this technique involves in situ splitting of the liver along the falciform ligament, and it is performed in conjunction with ligation of the right portal vein and portal vein branches to segment IV. The patient remains hospitalized after the first stage and 7-14 days postoperatively a CT scan is performed to evaluate the extent of FLV augmentation. Assuming sufficient FLV growth is observed, the patient then undergoes repeat laparotomy and extended right hepatectomy. Complications were observed in 16 of 25 patients $(64 \%)$ and mortality occurred in 3 of 25 patients $(12 \%)$. Biliary leakage occurred in 7 of 25 patients, 5 were managed using percutaneous techniques and 2 required re-exploration for leakage from the hepaticojejunostomy. At a median time of 9 days, a median increase in RLV of $74 \%$ was observed and there were no cases of irreversible liver failure.

The greatest advantage of ALPPS is its ability to induce rapid and robust hyperplasia in the future liver remnant. Because the time required to achieve maximal FLV is shorter than PVE, proponents of ALPPS feel that there may be a decrease in the potential for continued tumor growth during the regenerative phase. Furthermore, the technical difficulty of the second stage is reduced since the degree of adhesiolysis required is reduced compared to staged resections performed in conjunction with PVE. Some have also advanced the idea that for synchronous lesions simultaneous resection of colorectal primary tumors may be undertaken during the first stage of ALPPS [13]. Critics argue that the compacted timeline of ALPPS relative to the traditional 2-stage procedure facilitated by PVE inhibits the identification of patients with unfavorable tumor biology. Thus, patients who would have been spared an operation because they had growth of CRLM in the proposed liver remnant after PVE are now being subjected to an unnecessary operation. They also argue that a similar degree of liver hypertrophy can be achieved with PVE without the relatively high complication and mortality rate reported in the initial study by Schnitzbauer. The rate of complications, particularly the rate of bile fistulae has been a cause for concern among critics [14, 15]. At present, experience with this procedure is limited, and questions remain regarding oncologic outcomes and safety. Further study is necessary to determine the role of ALPPS in treating CRLM.

\section{Disappearing Liver Metastases}

In many centers, pre-hepatectomy chemotherapy has been promoted for the treatment of resectable CRLM. The rationale for this strategy is that the efficacy of modern chemotherapeutic regimens allows for tumor downsizing, greater preservation of liver parenchyma and, in a small group of patients, even a complete clinical response to chemotherapy. In addition, adjuvant approaches are plagued by high rates of omission of postoperative chemotherapy secondary to difficult and protracted recoveries from surgery. Disappearing liver metastases (DLM) refer to lesions that become radiographically occult on posttreatment imaging [16-21]. CLRM most likely to progress to DLM are those that are less than $2 \mathrm{~cm}$, as well as lesions treated with extended courses of chemotherapy $(7.7$ cycles for DLM vs. 5.5 cycles for tumors detectable on preoperative imaging) [19-21]. In fact, every additional round of neoadjuvant chemotherapy may increase the chance of DLM by $18 \%$ [19].

Though a complete radiologic response can occur, the rate at which this is observed is low. For example, DLM were noted in only 4 of 171 (3\%) patients who received pre-hepatectomy chemotherapy in the European Organization for the Research and Treatment of Cancer 40983 study [22]. Similarly, Adam et al. [1] found in a retrospective review of 767 patients that only $2(0.3 \%)$ had a complete clinical response. Though DLM are observed only in a small number of patients, the concept that even some patients may achieve a complete response of CRLM with chemotherapy alone is an appealing one, as it would avoid theoretically unnecessary resections [23, 24]. 
Imaging and the Problem of Disappearing Liver Metastases

Determining whether a complete clinical response represents a true pathologic complete response, however, is a difficult and imprecise task that ultimately rests on the sensitivity of cross-sectional imaging. Monitoring of response to chemotherapy is typically carried out with serial computed tomography scans (CT) and fluorodeoxyglucose positron emission tomography (FDG-PET). Technological improvements have made CT a valuable tool in following CRLM, and it has a sensitivity of 70-90\% and specificity of 85-90\% [2]. Despite improvements, however, CT has limitations especially following treatment with contemporary chemotherapy, and thus some DLM may actually represent undetected, viable metastases. Steatohepatitis, steatosis and other parenchymal effects that can arise as a result from chemotherapy can further compromise the ability of CT to detect subcentimeter CRLM $[2,25]$.

FDG-PET is another commonly used modality to assess the extent of the disease and to monitor the response of CRLM to chemotherapy. In the context of state-of-the-art CT and MRI, the principal value of PET lies in identifying extrahepatic metastatic disease. The loss or reduction of FDG-PET avidity correlates with the other measures of response to treatment (size, morphology), however, its utility as a surrogate for complete pathologic response of CRLM is limited by several factors. The sensitivity of FDG-PET is greatly reduced as the tumor size decreases and its sensitivity in detecting tumors less than $1 \mathrm{~cm}$ is poor [26]. Furthermore, tumors (especially those with central necrosis) can often display heterogeneous FDG uptake. The thin rim of FDG-avid tumor at the periphery may be difficult to detect and distinguish from background activity in the normal hepatic parenchyma [27]. Finally, systemic chemotherapy reduces glucose uptake of hepatic tumors relative to those not treated with chemotherapy, thus decreasing the sensitivity of FDG-PET [28].

MRI has a higher sensitivity and specificity in comparison with CT and FDG-PET, particularly in the evaluation of subcentimeter tumors [29, 30]. MRI has a particular advantage in imaging livers with chemotherapy-induced steatosis or steatohepatitis as fat-suppressing techniques can be used to improve detection of small lesions. In a meta-analysis performed by van Kessel et al. [31•], the pooled sensitivity of MRI for imaging CRLM after preoperative chemotherapy was 85.7 per cent (vs. $69.9 \%$ for CT, $54.5 \%$ for PET and $51.7 \%$ for PET-CT). Auer et al. [21] reported that failure to use pre-hepatectomy MRI resulted in more lesions being identified at the time of surgery after being incorrectly labeled DLM on preoperative cross-sectional imaging. Furthermore, disappearance of a lesion on preoperative MRI was associated with a greater likelihood of having a complete pathological response [21]. The current data suggest that MRI is the most appropriate imaging modality for assessment of patients with CRLM treated with preoperative chemotherapy, particularly DLM as some that are not seen on CT will be detected by MRI [32, 33].

What is the Meaning of Disappearing Liver Metastases?

A number of studies have shown that there is discordance between complete clinical response and complete pathological response. Tan et al. [27] examined loss of PET positivity of 34 lesions in 14 patients treated with prehepatectomy chemotherapy. On microscopic examination of these lesions, $85 \%(n=29)$ still had viable tumor despite the loss of FDG-PET avidity. Similarly, using CT as the primary imaging modality, Benoist et al. [20] found that 20 of 66 DLM actually had gross evidence of disease at the time of surgery. An additional 15 DLM sites that did not have any evidence of gross disease at the time of surgery were resected as well, and on microscopic evaluation 12 of 15 sites had viable tumor cells visible. The remaining 31 DLM sites that were not visible at the time of surgery were left in situ, and within 1 year 23 of 31 sites had evidence of recurrence. In total, persistent macroscopic, microscopic, or early recurrent disease was observed in $83 \%$ of DLM. Though the rate of durable remission and complete pathological response reported by van Vledder et al. [19] were somewhat higher than Benoist et al., there was still a significant discordance between complete clinical response and complete pathological response. Of the 126 DLM observed, $69(54.7 \%)$ were detected during surgery and treated either by resection $(n=55)$ or ablation $(n=14)$. Of the 55 DLM that were detected and resected, 19 lesions (34.5\%) showed a complete pathological response. Among 12 DLM not detected during surgery but included as part of hemi-hepatectomies, complete pathological response was observed in 7 (58.3\%). The remaining 45 DLM were left untreated in situ, and at a median follow-up of 20 months, $24(53.3 \%)$ did not show evidence of recurrence. Therefore, a true complete response was observed in 50 of the 112 DLM (44.6\%). It should be noted that MRI was not used routinely in either the Benoist $(0 \%)$ or the van Vledder $(13 \%)$ series. The lack of optimal post-chemotherapy/pre-hepatectomy imaging may have resulted in a higher number of missed lesions.

Some series of DLM have reported higher rates of complete pathological response largely because of liberal use of MRI and treatment with hepatic artery infusion (HAI) pumps [16-18, 21]. Elias et al. [16, 17], for example, 
reported a complete response rate of $86 \%$ among patients receiving HAI therapy prior to surgery versus $22 \%$ for those receiving systemic chemotherapy alone. Additionally, durable response was significantly associated with the use of HAI postoperatively for DLM that were not identified intraoperatively or resected. Similarly, Auer et al. [21] found that treatment with HAI was independently associated with the true complete response (odds ratio 6.2, $P=0.02)$

\section{Strategic Management of DLM}

Given the finding that complete pathological responses are not routinely associated with complete radiographic responses, the first strategy in managing DLM is to avoid them. Complete radiographic/clinical responses can in some cases be detrimental to surgical planning, as identification of the precise site of CRLM becomes difficult, complicating the attempts to achieve a negative resection margin. This is particularly true for small lesions located deep within the liver and for locations that would necessitate much larger resections to ensure that an occult site is removed. Thus, the goal of preoperative chemotherapy should be primarily to convert unresectable CRLM to resectable CRLM (and perhaps to better select these patients). The goal should not be to achieve maximal radiologic response, as the extended chemotherapy has the potential disadvantage of masking small lesions and decreasing the chance that they are successfully resected. When chemotherapy in these occasions must be delivered pre-hepatectomy, some have advocated for having fiducials implanted into higher risk masses that would be visible on intraoperative ultrasound at a later date. In patients with initially resectable CRLM, up-front resection should be considered prior to chemotherapy as a viable strategy, particularly if the size of CRLM is small. In patients with synchronous CRLM and asymptomatic primary tumors, a liver-first approach can be utilized to resect tumors in precarious locations.

In general, surgical extirpation is recommended for DLM in patients treated with systemic chemotherapy because only $20-40 \%$ of such lesions represent a true complete pathological response. Even for DLM treated with HAI, where the complete pathological response rate may be as high as $60-86 \%$, concordance between complete clinical and pathological response is not uniform [2, 25]. It should be noted that though intrahepatic recurrence of CRLM is higher for untreated DLM left in situ, there is no clear decrease in overall survival [19]. Nevertheless, the goal of hepatectomy, regardless of mode of chemotherapy, should be to resect all sites of CRLM that were present pre-treatment.

Intraoperative ultrasonography is a valuable tool that can help identify DLM at the time of surgery, but is highly dependent on operator skills. Systematic exploration of the liver leads to increase in the intraoperative detection rate of DLM, and the majority of these DLM identified by intraoperative ultrasound will have viable tumor on final microscopic examination [34]. Ferrero et al. reported the results of 67 DLM in 33 patients taken to the operating room for planned resection. At the time of laparotomy, 45 of 67 lesions $(67 \%)$ were identified; $6(9 \%)$ were grossly detectable and $39(58 \%)$ were identified using intraoperative ultrasound. Intraoperative detection of DLM by ultrasound was independently associated with the presence of viable tumor on microscopy $(P=0.001)$.

While DLM identifiable at the time of surgery should be removed clearly when in a resectable pattern, surgical decision-making becomes more complicated when the distribution of disease is such that DLM cannot be all resected in one setting. This is further complicated when DLM cannot be identified at the time of surgery. Several strategies exist if resection of all original sites is not feasible:

1. Extirpation of residual macroscopic disease and identifiable DLM can be performed, while leaving unidentifiable DLM untreated. The DLM left in situ should be followed with either close surveillance or adjuvant therapy (systemic chemotherapy or HAI therapy).

2. DLM can be treated with further systemic chemotherapy with or without HAI.

3. DLM can be tested prior to surgery with a chemotherapy break to better evaluate which DLM represent true pathologic responses, as those that are not will generally recur by a median time of 3-8 months $[19,21]$.

\section{Conclusions}

The current era of treatment for patients with colorectal cancer metastatic to the liver is flushed with challenges and nuanced care decisions. Tight communication between medical oncologists, hepatic and colorectal surgeons, and radiation oncologists is necessary to achieve optimal outcomes in these patients. Achieving surgical resection is central to optimal outcomes and as such the surgeon must play an active role in the decision-making to ensure the appropriate timing of surgical intervention.

\section{Compliance with Ethics Guidelines}

Conflict of Interest Kevin N. Shah and Bryan M. Clary declare that they have no conflict of interest.

Human and Animal Rights and Informed Consent This article does not contain any studies with human or animal subjects performed by the authors. 


\section{References}

Recently published papers of particular interest have been highlighted as:

- Of importance

1. Adam R, Wicherts DA, de Haas RJ, et al. Complete pathologic response after preoperative chemotherapy for colorectal liver metastases: myth or reality? J clin oncol. 2008;26(10):1635-41.

2. Bischof DA, Clary BM, Maithel SK, et al. Surgical management of disappearing colorectal liver metastases. $\mathrm{Br} \mathrm{j}$ surg. 2013; 100(11):1414-20.

3. Pawlik TM, Schulick RD, Choti MA. Expanding criteria for resectability of colorectal liver metastases. Oncologist. 2008;13(1):51-64.

4. Clavien PA, Petrowsky H, DeOliveira ML, et al. Strategies for safer liver surgery and partial liver transplantation. N Engl J Med. 2007;356(15):1545-59.

5. - Shindoh J, Vauthey JN, Zimmitti G, et al. Analysis of the efficacy of portal vein embolization for patients with extensive liver malignancy and very low future liver remnant volume, including a comparison with the associating liver partition with portal vein ligation for staged hepatectomy approach. Journal of the American College of Surgeons 2013;217(1):126-133; discussion 133-124. Shindoh and colleagues report the results of 141 patients who underwent PVE to augment FLV prior to hepatectomy. They compared morbidity and resectability rates in these patients to published reports of ALPPS and found that oncologic and technical results of PVE compare favorably with those of ALPPS.

6. Abdalla EK, Hicks ME, Vauthey JN. Portal vein embolization: rationale, technique and future prospects. Br J surg. 2001;88(2): 165-75.

7. Madoff DC, Abdalla EK, Gupta S, et al. Transhepatic ipsilateral right portal vein embolization extended to segment IV: improving hypertrophy and resection outcomes with spherical particles and coils. J Vasc Interv Radiol. 2005;16(2 Pt 1):215-25.

8. Farges O, Belghiti J, Kianmanesh R, et al. Portal vein embolization before right hepatectomy: prospective clinical trial. Ann Surg. 2003;237(2):208-17.

9. Shindoh J, Truty MJ, Aloia TA, et al. Kinetic growth rate after portal vein embolization predicts posthepatectomy outcomes: toward zero liver-related mortality in patients with colorectal liver metastases and small future liver remnant. J Am Coll Surg. 2013;216(2):201-9.

10. • Fischer C, Melstrom LG, Arnaoutakis D, et al. Chemotherapy after portal vein embolization to protect against tumor growth during liver hypertrophy before hepatectomy. JAMA surgery 2013;148(12):1103-1108. Fischer and colleagues report a retrospective review of patients who underwent $P V E$ and find that chemotherapy does not retard growth of the liver after PVE and may prevent cancer progression.

11. Jaeck D, Oussoultzoglou E, Rosso E, et al. A two-stage hepatectomy procedure combined with portal vein embolization to achieve curative resection for initially unresectable multiple and bilobar colorectal liver metastases. Annals of surgery 2004; 240(6):1037-1049; discussion 1049-1051.

12. - Schnitzbauer AA, Lang SA, Goessmann H, et al. Right portal vein ligation combined with in situ splitting induces rapid left lateral liver lobe hypertrophy enabling 2-staged extended right hepatic resection in small-for-size settings. Annals of surgery 2012;255(3):405-414. Schnitzbauer and colleagues report their experience with ALPPS and demonstrate the rapid growth of the FLR that can be seen with this technique.
13. de Santibanes E, Clavien PA. Playing Play-Doh to prevent postoperative liver failure: the "ALPPS" approach. Ann Surg. 2012;255(3):415-7.

14. Narita M, Oussoultzoglou E, Ikai I, et al. Right portal vein ligation combined with in situ splitting induces rapid left lateral liver lobe hypertrophy enabling 2-staged extended right hepatic resection in small-for-size settings. Annals of surgery 2012; 256(3):e7-8; author reply e16-17.

15. Aloia TA, Vauthey JN. Associating liver partition and portal vein ligation for staged hepatectomy (ALPPS): what is gained and what is lost? Annals of surgery 2012;256(3):e9; author reply e1619.

16. Elias D, Goere D, Boige V, et al. Outcome of posthepatectomymissing colorectal liver metastases after complete response to chemotherapy: impact of adjuvant intra-arterial hepatic oxaliplatin. Ann Surg Oncol. 2007;14(11):3188-94.

17. Elias D, Youssef O, Sideris L, et al. Evolution of missing colorectal liver metastases following inductive chemotherapy and hepatectomy. J Surg Oncol. 2004;86(1):4-9.

18. Tanaka K, Takakura H, Takeda K, et al. Importance of complete pathologic response to prehepatectomy chemotherapy in treating colorectal cancer metastases. Ann Surg. 2009;250(6):935-42.

19. van Vledder MG, de Jong MC, Pawlik TM, et al. Disappearing colorectal liver metastases after chemotherapy: should we be concerned? J Gastrointest Surg. 2010;14(11):1691-700.

20. Benoist S, Brouquet A, Penna C, et al. Complete response of colorectal liver metastases after chemotherapy: does it mean cure? J clin oncol. 2006;24(24):3939-45.

21. Auer RC, White RR, Kemeny NE, et al. Predictors of a true complete response among disappearing liver metastases from colorectal cancer after chemotherapy. Cancer. 2010;116(6):1502-9.

22. Nordlinger B, Sorbye H, Glimelius B, et al. Perioperative chemotherapy with FOLFOX4 and surgery versus surgery alone for resectable liver metastases from colorectal cancer (EORTC Intergroup trial 40983): a randomised controlled trial. Lancet. 2008;371(9617):1007-16.

23. Gruenberger B, Scheithauer W, Punzengruber R, et al. Importance of response to neoadjuvant chemotherapy in potentially curable colorectal cancer liver metastases. BMC Cancer. 2008;8:120.

24. Mentha G, Majno P, Terraz S, et al. Treatment strategies for the management of advanced colorectal liver metastases detected synchronously with the primary tumour. Eur J Surg Oncol. 2007;33(Suppl 2):S76-83.

25. Adams RB, Aloia TA, Loyer E, et al. Selection for hepatic resection of colorectal liver metastases: expert consensus statement. HPB : the official journal of the International Hepato Pancreato Biliary Association 2013;15(2):91-103. AHPBA, SSO and SSAT consensus statement on selection of patients for resection of CRLM including radigraphic findings, anatomic considerations and management of DLM.

26. Fong Y, Saldinger PF, Akhurst T, et al. Utility of $18 \mathrm{~F}-\mathrm{FDG}$ positron emission tomography scanning on selection of patients for resection of hepatic colorectal metastases. Am J Surg. 1999;178(4):282-7.

27. Tan MC, Linehan DC, Hawkins WG, et al. Chemotherapyinduced normalization of FDG uptake by colorectal liver metastases does not usually indicate complete pathologic response. J Gastrointest Surgery. 2007;11(9):1112-9.

28. Akhurst T, Kates TJ, Mazumdar M, et al. Recent chemotherapy reduces the sensitivity of $[18 \mathrm{~F}]$ fluorodeoxyglucose positron emission tomography in the detection of colorectal metastases. J Clin Oncol. 2005;23(34):8713-6.

29. Fowler KJ, Linehan DC, Menias CO. Colorectal liver metastases: state of the art imaging. Ann Surg Oncol. 2013;20(4):1185-93. 
30. Niekel MC, Bipat S, Stoker J. Diagnostic imaging of colorectal liver metastases with CT, MR imaging, FDG PET, and/or FDG PET/CT: a meta-analysis of prospective studies including patients who have not previously undergone treatment. Radiology. 2010; 257(3):674-84.

31. - van Kessel CS, Buckens CF, van den Bosch MA, et al. Preoperative imaging of colorectal liver metastases after neoadjuvant chemotherapy: a meta-analysis. Annals of surgical oncology 2012;19(9):2805-2813. Meta-analysis of imaging modalities in the setting of neoadjuvant chemotherapy. MRI appears to be the most sensitive imaging modality for preoperative assessment of patients with CRLM, followed by CT.
Diagnostic accuracy of PET and PET-CT is strongly affected by chemotherapy.

32. Charnsangavej C, Clary B, Fong Y, et al. Selection of patients for resection of hepatic colorectal metastases: expert consensus statement. Ann Surg Oncol. 2006;13(10):1261-8.

33. Smith JT, Ward J, Guthrie JA, et al. Detection of colorectal metastases in patients being treated with chemotherapy utilising SPIO-MRI: a radiological-pathological study. Magn Reson Imaging. 2012;30(10): 1446-53.

34. Ferrero A, Langella S, Russolillo N, et al. Intraoperative detection of disappearing colorectal liver metastases as a predictor of residual disease. J Gastrointest Surg. 2012;16(4):806-14. 